\title{
DEFORMATIONS OF CLOSED SPACE CURVES
}

\author{
E. A. FELDMAN
}

\section{Introduction}

In this note we will be interested in closed space curves, that is $C^{k}(k \geq 2)$ immersions of $S^{1}$ into $R^{3}$. We say a space curve is non-degenerate if the square of the curvature is never zero. This non-degeneracy condition is the classical hypothesis used to insure the existence of the moving Frenet frame along the curve. The question we would like to ask is the following one. Given any two closed non-degenerate space curves, when are they homotopic through a homotopy composed entirely of closed non-degenerate space curves? More precisely we want to study the space $N$ of non-degenerate closed space curves, considered as a subspace of $C^{k}\left(S^{1}, R^{3}\right)$, the $C^{k}$ maps from $S^{1}$ into $R^{3}$, with the $C^{k}$-topology $(k \geq 2)$ [4]. We ask: what are the arc components of $N$ ? A continuous path in $N$ will be called a non-degenerate homotopy.

It will be convenient to make all homotopies based. To make this specific let us first define the Frenet frame for $\gamma(s) \in N$. This is done by fixing the sign of the curvature to be positive, and letting the principal normal $t_{2}(s)$ be defined by $d t_{1}(s) / d s=k(s) t_{2}(s)$, where $s$ is arc-length parameter, $t_{1}(s)=d \gamma(s) / d s$ is the unit tangent vector, and $k(s)$ is the curvature of the given space curve $\gamma$. One then defines the binormal vector $t_{3}(s)$ by the formula $t_{3}(s)=t_{1}(s) \times t_{2}(s)$. Now let us fix a base point $\theta_{0} \in S^{1}$, and let

$$
N_{0}=\left\{\gamma \in N \mid \gamma\left(\theta_{0}\right)=0, t_{1}\left(\theta_{0}\right)=e_{1}, t_{2}\left(\theta_{0}\right)=e_{2}, t_{3}\left(\theta_{0}\right)=e_{3}\right\},
$$

where $\left(t_{1}, t_{2}, t_{3}\right)$ is the Frenet frame of $\gamma$, and the $e_{i}$ are the unit coordinate vectors of $R^{3}$ (i.e., $e_{1}=(1,0,0)$ etc.). An element of $N_{0}$ will be called a based non-degenerate curve, and a continuous path in $N_{0}$ a based non-degenerate homotopy. By using rotations and translations the arc components of $N$ are determined by those of $N_{0}$, because the group of rigid motions is connected. Given any $\gamma \in N_{0}$, we define $F(\gamma): S^{1} \rightarrow S O(3)$, by associating to each point of $\gamma$, its Frenet frame, where $S O(3)$ is the special orthogonal group. We see $F(\gamma)$ is of class $C^{k-2}$, and $F(\gamma)\left(\theta_{0}\right)=f_{0}=\left(e_{1}, e_{2}, e_{3}\right) \in S O(3)$. Our main result is the following theorem.

Communicated by J. Eells, Jr., November 17, 1967. This research was partially supported by NSF grant GP-5227. 
Theorem I. Let $\gamma$ and $\eta \in N_{0}$. Then $\gamma$ and $\eta$ are based non-degenerately homotopic if and only if $F(\gamma)$ is based homotopic to $F(\eta)$ (that is they determine the same element of $\left.\pi_{1}\left(S O(3), f_{0}\right)\right)$.

The "if" part is obvious; hence the main task will be to show the converse. We will now apply the theorem to get the desired classification.

It is well known that $\pi_{1}\left(S O(3), f_{0}\right) \cong Z_{2}$ and that the generator is represented by the curve of 3-frames $f(\theta)=\left(f_{1}(\theta), f_{2}(\theta), e_{3}\right)$ where $f_{1}(\theta)=(\cos \theta, \sin \theta, 0)$, and $f_{2}(\theta)=(-\sin \theta, \cos \theta, 0)$ for $0 \leq \theta \leq 2 \pi$ (see [6]). Let $\alpha(\theta)=(\sin \theta$, $1-\cos \theta, 0)$. Then $\alpha \in N_{0}$, and $F(\alpha)(\theta)=f(\theta)$. Therefore we see $N_{0}$ has two connected components, the first determined by traversing the circle $\alpha$ once, the second by traversing $\alpha$ twice (i.e., by $\alpha(2 \theta), 0 \leq \theta \leq 2 \pi$ ). Hence, if we deform the latter curve a bit so it is an embedded circle, we see that any $\gamma \in N\left(N_{0}\right)$ is (based) non-degenerately homotopic to either the circle or curve 2 pictured below.

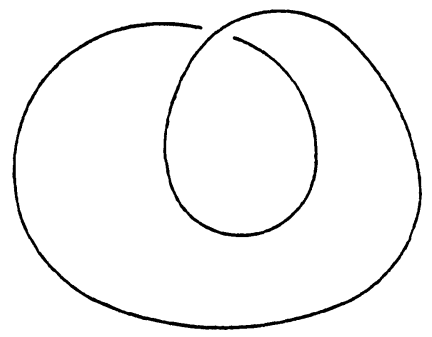

Curve 2

Besides the intrinsic geometric interest of this problem, it is also the most elementary open part of the following more general problem. Let $X$ be a Riemanian manifold. We say an immersed circle is non-degenerate if the geodesic curvature never vanishes. When are two non-degenerate immersed circles homotopic through non-degenerate immersions? Let us restrict ourselves to curves which are "based", that is, fix $\theta_{0} \in S^{1}, x_{0} \in X, f_{1}$ and $f_{2}$ mutually perpendicular unit tangent vectors at $x_{0}$, and restrict ourselves to $f: S^{1} \rightarrow X$, such that $f\left(\theta_{0}\right)=x_{0}$, and the unit tangent (unit principal normal) to $f$ at $\theta_{0}$ is $f_{1}\left(f_{2}\right)$. By associating with each $\theta \in S^{1}$ the unit tangent and unit principal normal of $f$ at $f(\theta)$ one obtains a curve in $V_{2}(X)$ the orthonormal two frame bundle of $X$. Hence the non-degenerate immersion $f$ defines an element $V(f)$ of $\pi_{1}\left(V_{2}(X), f_{0}\right)$, where $f_{0}=\left(x_{0}, f_{1}, f_{2}\right)$. If $f$ and $g$ are based nondegenerate immersions of $S^{1}$ in $X$, is the condition that $V(f)=V(g)$ sufficient to insure that $f$ is based nondegenerate regularly homotopic to $g$ ? When $\operatorname{dim} X \geq 4$ it is indeed the case since $\pi_{1}\left(V_{2, k}\right)=0(k \geq 4)$ where $V_{2, k}$ is the Stiefel manifold of 2 frames in $k$-space. Therefore $\pi_{1}\left(V_{2}(X), f_{0}\right) \cong \pi_{1}\left(X, x_{0}\right)$. We showed in [1] that in this case $f$ and $g$ are (based) non-degenerately homotopic if and only if they were (based) homotopic. This leaves only two 
and there dimensional manifolds, and this note supplies an affirmative answer to the question in case $X=R^{3}$, since $V_{2,3} \cong S O(3)$. For $X=R^{2}$, the question is quite easily answered. The winding number (normal degree) is the only invariant, and every winding is realized except of course 0 . The proof is much easier than that of the Whitney-Graustein theorem [8], since the positivity (negativity) of the curvature guarantees that the tangent map is a covering map of $S^{1}$ by $S^{1}$. If we take the above question as a conjecture I would assume it is true for $\operatorname{dim} X=3$, and false for $\operatorname{dim} X=2$. One can clearly ask the analogous questions which arise by demanding that higher and higher order curvatures never vanish.

Finally I would like to thank R. Sacksteder for making an essential simplification in the proof of Fenchel's theorem [3], and C. Weaver for pointing out a way of simplifying the original proof of this theorem.

\section{The factorization and spherical curves}

Let us first identify $S O(3), V_{2,3}$ and $T_{0}\left(S^{2}\right)$, the unit tangent circle bundle of $S^{2}$, as follows. Note for any 3-frame $\left(f_{1}, f_{2}, f_{3}\right) \in S O(3), f_{3}=f_{1} \times f_{2}$, and $\left(f_{1}, f_{2}\right) \in V_{2,3}$. Thus we can identify $V_{2,3}$ and $S O(3)$. We then view $\left(f_{1}, f_{2}\right) \in V_{2,3}$ as defining a point $f_{1} \in S^{2}$, and a unit tangent vector to $S^{2}$ at $f_{1}$ by parallel translating the foot of $f_{2}$ from 0 to $f_{1}$. With the above identifications in mind we will use these three spaces interchangeably. Now let us fix $\theta_{0} \in S^{1}$, and $f_{0}=\left(e_{1}, e_{2}\right) \in V_{2,3}$, where $e_{i}$ is the $i$-th coordinate vector. Let $V_{0}=\left\{f \in C^{k-2}\right.$ $\left.\left(S^{1}, V_{2,3}\right) \mid f\left(\theta_{0}\right)=f_{0}\right\}$. Then by associating to each $\gamma \in N_{0}$, the moving 2-frame defined by its unit tangent and principal normal vectors, we have defined a

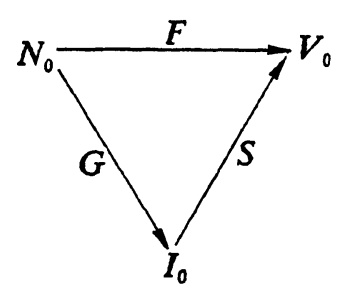
continuous map $F: N_{0} \rightarrow V_{0}$, where $V_{0}$ is given the $C^{k-2}$ topology. Let $I_{0}=\left\{\lambda \in C^{k-1}\left(S^{1}, S^{2}\right) \mid \lambda\right.$ is an immersion, $\lambda\left(\theta_{0}\right)=e_{1}$, and $\left.\lambda^{\prime}\left(\theta_{0}\right) /\left|\lambda^{\prime}\left(\theta_{0}\right)\right|=e_{2}\right\}$, and give $I_{0}$ the $C^{k-1}$ topology. Then we can factor $F$ through $I_{0}$ as follows. Let $\gamma \in N_{0}$, and set $G(\gamma)(\theta)=\gamma^{\prime}(\theta) /\left|\gamma^{\prime}(\theta)\right|$. We could define $G(\gamma)$ for an arbitrary $C^{1}$ space curve $\gamma$; however $G(\gamma)$ is an immersion if and only if $\gamma$ is non-degenerate. $G$ clearly maps $N_{0}$ into $I_{0}$ continuously. Let $\lambda \in I_{0}$, and set $S(\lambda)(\theta)=\left(\lambda(\theta), \lambda^{\prime}(\theta) /\left|\lambda^{\prime}(\theta)\right|\right)$. Then $S$ is continuous and $F=S \circ G$. We now quote the following theorem of Smale [5].

Theorem S. Let $\lambda$ and $\bar{\lambda} \in I_{0}$. Then $\lambda$ and $\bar{\lambda}$ are based regularly homotopic (i.e. lie in the same arc component of $I_{0}$ ), if and only if $S(\lambda)$ and $S(\bar{\lambda})$ represent the same element of $\pi_{1}\left(V_{2,3} ; f_{0}\right)$. If $S(\lambda)$ is homotopic to $S(\bar{\lambda})$, and furthermore there exists a neighborhood $U$ of $\theta_{0}$ on which $\lambda$ and $\bar{\lambda}$ agree, then we can find a neighborhood $U^{\prime} \subset U$ of $\theta_{0}$, and a regular homotopy $\lambda_{s}$ joining $\lambda$ to $\bar{\lambda}$ (i.e. a path in $I_{0}$ ) such that $\lambda_{s}$ and $\lambda$ agree on $U^{\prime}$ for $0 \leq s \leq 1$.

Remark. This is a special case of Smale's theorem and it admits a rather easy elementary proof, by first applying the Whitney-Graustein theorem [8] 
to the punctured sphere, and then "cancelling" loops. By applying theorem $S$ we see that the following Theorem $I^{\prime}$ is equivalent to Theorem I.

Theorem I'. Let $\gamma$ and $\bar{\gamma} \in N_{0}$. Then $\gamma$ is based non-degenerately homotopic to $\bar{\gamma}$ if and only if $G(\gamma)$ is based regularly homotopic to $G(\bar{\gamma})$.

If we are to prove $I^{\prime}$, we have to know which closed spherical curves are tangent indicatrices of closed space curves. This question is answered by the following proposition of Fenchel [2], [3]. In the "if" part we will merely sketch Loewner's well known remarks [2]. In the "only if" part Fenchel's argument is somewhat simplified by a remark of Sacksteder; we include this proof because the argument is central in the proof of Theorem $\mathrm{I}^{\prime}$.

Let us fix the following notation. If $A$ is a subset of $R^{3}$, let $[A], A^{c}, A^{i}$ denote respectively the convex hull of $A$, the closure of $A$, and the interior of $A$.

Proposition 2.1. a) Let $\gamma(\theta)$ be a closed non-plane space curve of class $C^{k}(k \geq 1)$, and $G(\theta)=\gamma^{\prime}(\theta) /\left|\gamma^{\prime}(\theta)\right|$ the tangent map. Then $0 \in\left[\left\{G(\theta) \mid \theta \in S^{1}\right\}\right]^{i}$.

b) Let $\lambda(\theta)$ be a closed curve of class $C^{k-1}(k \geq 1)$ on $S^{2} \subseteq R^{3}$. If $0 \in\left[\left\{\lambda(\theta) \mid \theta \in S^{1}\right\}\right]^{i}$ then $\lambda(\theta)$ is the tangent indicatrix of a closed $C^{k}$, nonplane, space curve.

Proof. a) Let $P$ be a plane in $R^{3}$ which does not meet $\gamma$. Then there must be both a maximum and a minimum of the distance from $P$ to $\gamma$. If $p_{*}=r\left(\theta_{*}\right)$ is either a maximum or minimum point of this distance function, then $G\left(\theta_{*}\right)$ is parallel to $P$ and therefore $G\left(\theta_{*}\right)$ lies on the great circle determined by the intersection of $S^{2}$ with the plane parallel to $P$ which passes through the origin. The fact that we have both maxima and minima implies that the set $\left\{G(\theta) \mid \theta \in S^{1}\right\}$ meets both open hemispheres determined by the aforementioned great circle.

b) Let $\lambda(\theta)$ be viewed as a periodic vector valued function of period $2 \pi$, $C$ be the vector space of real valued $C^{\infty}$ functions on $S^{1}$, viewed as periodic functions with period $2 \pi$, and $P=\{\rho \in C \mid \rho(\theta)>0,0 \leq \theta \leq 2 \pi\}$. We note that it suffices to find a $\rho \in P$ such that $\int_{0}^{2 \pi} \rho(\theta) \lambda(\theta) d \theta=0$, because we can then set $\gamma(t)=\int_{0}^{t} \rho(\theta) \lambda(\theta) d \theta$, and $\gamma$ will be the desired space curve.

Let $K=\left\{y \in R^{3} \mid\right.$ there exists $\rho \in P$ such that $\left.y=\int_{0}^{2 \pi} \rho(\theta) \lambda(\theta) d \theta\right\}$. We see that $K$ is a convex cone because $P$ is such. At this point Sacksteder noticed that it suffices to show $\{\lambda(\theta) \mid 0 \leq \theta \leq 2 \pi\} \subseteq K^{c}$, because $0 \in[\{\lambda(\theta) \mid 0 \leq \theta \leq 2 \pi\}]^{i}$, and therefore $0 \in K$ provided the above inclusion holds. The remainder of the argument is essentially Fenchel's.

Let

$$
\tilde{\varphi}(x)= \begin{cases}e\left(1 / x^{2}-1\right) & -1 \leq x \leq 1, \\ 0 & \text { elsewhere }\end{cases}
$$


where $e(t)=e^{t}$. Let $b=\int_{-\infty}^{\infty} \tilde{\varphi}(x) d x$; therefore we set $\varphi(x)=\frac{1}{b} \tilde{\varphi}(x)$. Pick $\theta_{*}$ such that $0 \leq \theta_{*} \leq 2 \pi$, and $\xi$ such that $0<2 \pi \xi<1$. We then define a

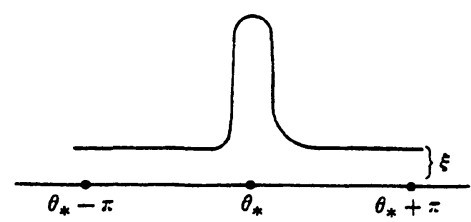
function $f_{\xi}\left(\theta, \theta_{*}\right)$ on an interval of length $2 \pi$ with $\theta_{*}$ as midpoint as follows. $f_{\xi}\left(\theta, \theta_{*}\right)$ $=\xi+h \varphi\left(\frac{\theta-\theta_{*}}{\xi}\right)$ where $h$ is so chosen that $\int_{\theta_{*}-\pi}^{\theta_{*}+\pi} f_{\xi}\left(\theta, \theta_{*}\right) d \theta=1$. We extend $f_{\xi}$ to

all of $R$ by making it periodic of period $2 \pi . f_{\xi}\left(\theta, \theta_{*}\right) \in P$. Let $\lambda_{\xi}\left(\theta_{*}\right)$ $=\int_{0}^{2 \pi} \lambda(\theta) f_{\xi}\left(\theta, \theta_{*}\right) d \theta$. Then $\lambda_{\xi}\left(\theta_{*}\right) \in K$. Note that $\int_{0}^{2=} f_{\xi}=1$ implies that

$$
\left|\lambda_{\xi}\left(\theta_{*}\right)-\lambda\left(\theta_{*}\right)\right|=\mid \int_{0}^{2 \pi}\left[\lambda(\theta)-\lambda\left(\theta_{*}\right)\right]\left(f_{\xi}\left(\theta, \theta_{*}\right) d \theta_{\vdots}^{\vdots} \cdot\right.
$$

Thus we see $\lambda_{\xi}\left(\theta_{*}\right) \rightarrow \lambda\left(\theta_{*}\right)$ as $\xi \rightarrow 0$ by the continuity of $\lambda$ and the construction of $f_{\xi}$. Hence $\lambda\left(\theta_{*}\right) \in K^{c}$.

We can prove Theorem $\mathrm{I}^{\prime}$, if we can carry out the following procedures. First, let $G_{0}=G\left(\gamma_{0}\right)$ and $G_{1}=G\left(\gamma_{1}\right)$ be the tangent images of $\gamma_{0}$ and $\gamma_{1} \in N_{0}$. If $G_{0}$ and $G_{1}$ are regularly homotopic, does there exist a base regular homotopy $G_{s}$ joining $G_{0}$ to $G_{1}$ such that $0 \in\left[\left\{G_{s}(\theta) \mid 0 \leq \theta \leq 2 \pi\right\}\right]^{i}$ for each $s$, $0<s<1$ ? Second, if we have the desired homotopy $G_{s}$ above, can we find a continuous 1-parameter family of weighting functions $\rho_{s} \in P$ such that $\int_{0}^{2 \pi} \rho_{s}(\theta) G_{s}(\theta) d \theta=0$ where $\rho_{0}(\theta)=\left|\gamma_{0}^{\prime}(\theta)\right|$ and $\rho_{1}(\theta)=\left|\gamma_{1}^{\prime}(\theta)\right|$ ?

\section{First deformations}

From now on we will view all maps with $S^{1}$ as source, as periodic maps of period $2 \pi$ with $R$ as source.

Proposition 3.1. Let $\gamma \in N_{0}$. Then there exists a based non-degenerate homotopy between $\gamma$ and an element $\bar{\gamma} \in N_{0}$, where $\bar{\gamma}$ has the following property: For some parametrization $t$ of $\gamma$, we can find a number $l>0$ depending on $\gamma$, such that $G(\bar{\gamma})(t)=(\cos t, \sin t, 0)$ for $0 \leq t \bmod 2 \pi \leq l$.

Proof. Let us note that $\gamma$ is non-degenerate if and only if the vectors $\gamma^{\prime}(t)$ and $\gamma^{\prime \prime}(t)$ are linearly independent for each $t, 0 \leq t \leq 2 \pi$. By reparametrizing $\gamma$ if necessary we can assume $\gamma^{\prime}(0)=e_{1}$, and $\gamma^{\prime \prime}(0)=\left|\gamma^{\prime \prime}(0)\right| e_{2}$ where $e_{i}$ is the unit vector in the $i$-th direction. If we let $\gamma(t)=\left(\gamma_{1}(t), \gamma_{2}(t), \gamma_{3}(t)\right)$, we see that there exists a number $l_{1}>0$ such that if $|t| \bmod 2 \pi<l_{1}$, then the vectors $\left(\gamma_{1}^{\prime}(t), \gamma_{2}^{\prime}(t), 0\right)$ and $\left(\gamma_{1}^{\prime \prime}(t), \gamma_{2}^{\prime \prime}(t), 0\right)$ are linearly independent. Let $\phi(t)$ be a $C^{\infty}$ periodic function of period $2 \pi$, such that $\psi(t) \equiv 1$ for $0 \leq|t| \bmod 2 \pi \leq l_{1} / 2$, 
$\phi(t) \equiv 0$ for $|t| \bmod 2 \pi \geq l_{1}$, and $0 \leq \psi \leq 1$. We define a based non-degenerate homotopy by the formula

$$
\gamma_{u}(t)=\left(\gamma_{1}(t), \gamma_{2}(t),(1-u \psi(t)) \gamma_{0}(t)\right)
$$

such that $\gamma_{0}(t)=\gamma(t)$, and $\gamma_{u}(t)=\gamma(t)$ for $|t| \bmod 2 \pi \geq l_{1}$. Then $\gamma_{u}(t) \in N_{0}$ by our choice of $l_{1}$. Let $\bar{\gamma}(t)=\gamma_{1}(t)$. For $0 \leq|t| \bmod 2 \pi \leq l_{1} / 2, \bar{\gamma}(t)$ lies in the $(x, y)$ plane and has positive curvature. Hence we can reparametrize $\bar{\gamma}$ so that $G(\bar{\gamma})(\tau)=(\cos \tau, \sin \tau, 0)$ for $0 \leq|\tau| \bmod 2 \pi \leq l$ for some $l>0$, which depends upon $l_{1}$.

Proposition 3.2. Let $\gamma(t) \in N_{0}$, and assume $\gamma$ lies in the $(x, y)$ plane. Then for an arbitrarily small $\xi, 0<\xi<\pi$, $\gamma$ is based non-degenerately homotopic to a nonplane curve $\eta$, such that $\gamma(t)=\eta(t)$ for $0 \leq|t| \bmod 2 \pi \leq \pi-\xi$.

Proof. Let $\varphi_{0}(t)=\left\{\begin{array}{cc}e\left(1 / t^{2}-1\right), & -1 \leq t \leq 1, \\ 0 & \text { elsewhere. }\end{array}\right.$ Extend $\varphi_{0}\left(\frac{t-\pi}{\xi}\right) \mid[0,2 \pi]$ for $0<\xi<\pi$ to all of $R$, by making it periodic of period $2 \pi$, and denote this function by $\varphi$. Let $\phi(u): R \rightarrow R$ by a $C^{\infty}$ function, such that $\phi(u)=0$ for $u \leq 0, \phi(u)=1$ for $u \geq 1$ and $0 \leq \psi(u) \leq 1$. Set $\gamma_{u}(t)=\left(\gamma_{1}(t), \gamma_{2}(t), \phi(u) \varphi(t)\right)$. Then $\gamma_{0}(t)=\gamma(t)$ and define $\eta(t)=\gamma_{1}(t)$. We thus see that $\gamma_{u}(t)$ is the desired non-degenerate homotopy.

Lemma 3.3. Let $\lambda(t)$ and $\eta(t)$ be in $I_{0}$, and assume that $\lambda$ and $\eta$ are based regularly homotopic and that

$$
0 \in[\{\lambda(t) \mid 0 \leq t \leq 2 \pi\}]^{i} \cap[\{\eta(t) \mid 0 \leq t \leq 2 \pi\}]^{i} .
$$

Finally we assume that $\lambda(t)=\eta(t)=(\cos t, \sin t, 0)$ for $0 \leq t \leq l_{1}$, for some $l_{1}>0, l_{1}<\pi$. Then there exists a based regular homotopy $\lambda_{u}(t)$ joining $\lambda$ to $\eta$ such that $0 \in\left[\left\{\lambda_{u}(t) \mid 0 \leq t \leq 2 \pi\right\}\right]^{i}$ for each $u, 0 \leq u \leq 1$.

Proof. First pick a regular homotopy $\nu_{u}(t)$ such that $\nu_{0}=\lambda, \nu_{1}=\eta$, and $\nu_{u}(t)=(\cos t, \sin t, 0)$ for $0 \leq t \leq l$ where $0<l<l_{1}$, and is determined by Theorem $S$ of $\S 2$. Let us look at the great circle $c$ which passes through $\lambda\left(\frac{1}{2} l\right)$ and the north pole. Let $\Omega_{u}(t)$ be a regular homotopy of immersions

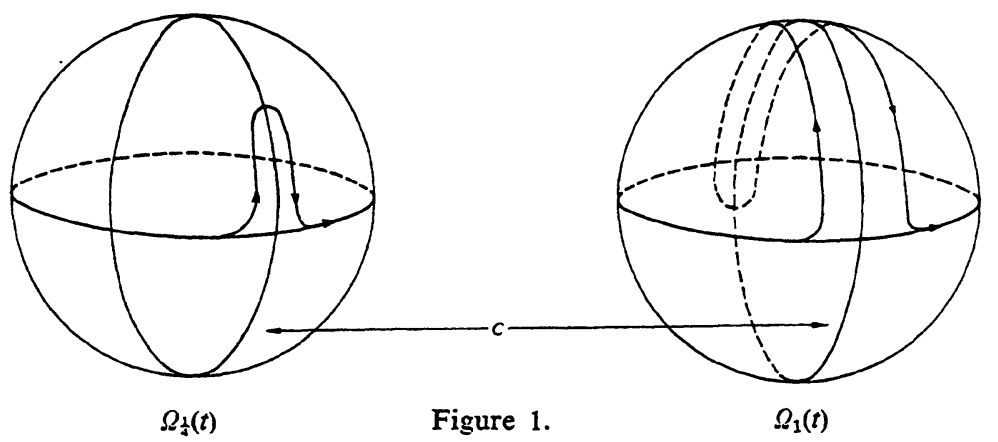


of the interval $[0, l]$ into $S^{2}$ which we will describe as follows. Let $\Omega_{u}(t)$ $=(\cos t, \sin t, 0)$ for $0 \leq t \leq \frac{1}{4} l$ and for $\frac{3 l}{4} \leq t \leq l$, and let $\Omega_{0}(t)=(\cos t$, $\sin t, 0$ ) for all $t$. Finally as $u$ varies from 0 to 1 we pull out a long skinny "bump" which is symmetric about the great circle $c$, until finally at $u=1$ the bump has gone more than halfway around the circle $c$, as we see in figure 1 .

We can now define the desired homotopy $\lambda_{u}(t)$.

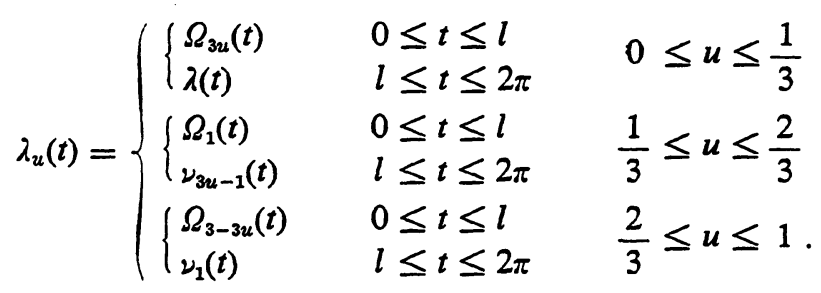

This homotopy clearly has the correct properties as the long hump guarantees the convexity property as we deform through the homotopy $\nu_{u}$.

\section{Deforming the weighting functions}

Let $P=\left\{\rho \in C^{k-1}\left(S^{1}, R\right) \mid \rho(\theta)>0\right.$ for $\left.0 \leq \theta \leq 2 \pi\right\}$. If we give $C^{k-1}\left(S^{1}, R\right)$ the $C^{k-1}$ topology, then $P$ is an open convex cone. Denote $C^{k-1}\left(S^{1}, R\right)$ by $C$.

Lemma 4.1. Let $\lambda_{0}$ and $\lambda_{1}$ be non-plane elements of $I_{0}$, such that for some $l>0,0 \leq \theta \leq l<\pi, \lambda_{0}(\theta)=\lambda_{1}(\theta)=(\cos \theta, \sin \theta, 0)=\alpha(\theta)$. Assume furthermore that

$$
0 \in\left[\left\{\lambda_{0}(\theta) \mid 0 \leq \theta \leq 2 \pi\right\}\right]^{i} \cap\left[\left\{\lambda_{1}(\theta) \mid 0 \leq \theta \leq 2 \pi\right\}\right]^{i},
$$

and that $\lambda_{0}$ is based regularly homotopic to $\lambda_{1}$. Pick $\rho_{i}(\theta) \in P, i=0,1$, such that $\int_{0}^{2 \pi} \rho_{i}(\theta) \lambda_{i}(\theta) d \theta=0$ for $i=0,1$. We can then find a regular homotopy $\lambda_{w}(\theta), 0 \leq w \leq 1$, between $\lambda_{0}$ and $\lambda_{1}$, an interval $\left[0, l_{1}\right], 0<l_{1}<l$, and a continuous path $\rho_{w}(\theta) \in P$ (continuous as a map from $[0,1]$ into $P$ ) with the following properties: $\lambda_{w}(\theta)=\alpha(\theta)$ for $0 \leq w \leq 1$ and $\theta \in\left[0, l_{1}\right]$, each $\lambda_{w}(\theta)$ is nonplaner, $0 \in\left[\left\{\lambda_{w}(\theta) \mid 0 \leq \theta \leq 2 \pi\right\}\right]^{i}$ for each $w$, and $\int_{0}^{2 \pi} \lambda_{w}(\theta) \rho_{w}(\theta) d \theta=0$ for each $w$.

Before proving Lemma 4.1 we note that this lemma and the results of $\S 3$ will imply theorem $I^{\prime}$ as follows. Let $\eta_{0}$ and $\eta_{1} \in N_{0}$, and assume $G\left(\eta_{0}\right)$ is based regularly homotopic to $G\left(\eta_{1}\right)$. By applying Propositions 3.1 and 3.2 we can find curves $\gamma_{i} \in N_{0}, i=0,1$, such that $\gamma_{i}$ is based non-degenerately homotopic to $\eta_{i}, i=0,1$, and such that the curves $\lambda_{i}=G\left(\gamma_{i}\right), i=0,1$, have the properties of the $\lambda_{i}$ of Lemma 4.1. Let $\rho_{i}(\theta)=\left|\gamma_{i}^{\prime}(\theta)\right|$, and $\lambda_{w}(\theta)$ and $\rho_{w}(\theta)$ be 
the regular homotopy and path of weighting functions determined by Lemma 4.1. We then set $\gamma_{w}(t)=\int_{0}^{t} \lambda_{w}(\theta) \rho_{w}(\theta) d \theta$ which determines the desired path in $N_{0}$.

Proof of Lemma 4.1. By Lemma 3.3 we can find a based regular homotopy $\lambda_{u}(\theta)$ joining $\lambda_{0}$ to $\lambda_{1}$, and an interval $\left[0, l_{1}\right], 0<l_{1}<l$, such that $0 \in\left[\left\{\lambda_{u}(\theta) \mid 0 \leq \theta \leq 2 \pi\right\}\right]^{i}$ for each fixed $u$, and such that $\lambda_{u}(\theta)=\alpha(\theta)$ for $\theta \in\left[0, l_{1}\right], 0 \leq u \leq 1$. Let $L=L^{2}\left(S^{1}, R\right)$ denote the space of square integrable periodic real-valued functions, and write $\lambda_{u}(\theta)=\left(\lambda_{u, 1}(\theta), \lambda_{u, 2}(\theta), \lambda_{u, 3}(\theta)\right)$. For each fixed $j, \lambda_{u, j} \in C \subseteq L$, and in fact $\lambda_{u, 1}, \lambda_{u, 2}$ and $\lambda_{u, 3}$ are linearly independent elements of $L$ as well as of $C$, for each $u$. We will now adopt the following notation. If $x(\theta)$ and $y(\theta) \in C$ we will set $\langle x(\theta), y(\theta)\rangle=\int_{0}^{2 \pi} x(\theta) y(\theta) d \theta$. We will suppress the circle variable $\theta$, and we will write $\lambda_{u, j}(\theta)$ as $\lambda_{j}(u)$ for $1 \leq j \leq 3$, and $\rho_{i}(\theta)$ as $\rho(i)$ for $i=1,2$. Hence we are given three continuous curves $\lambda_{j}(u)$ in $C$, which for each fixed $u$, determines 3 elements linearly independent in both $C$ and $L$. Therefore we want to find a curve $\rho(u)$ in $P$ joining $\rho(0)$ to $\rho(1)$ such that $\left\langle\rho(u), \lambda_{j}(u)\right\rangle=0$ for $j=1,2$ and 3 .

By the Gram-Schmidt process we can replace the curves $\left(\lambda_{j}(u)\right), 1 \leq j \leq 3$, by curves $\left(\mu_{j}(u)\right), 1 \leq j \leq 3$, such that $\left\langle\mu_{i}(u), \mu_{j}(u)\right\rangle=\delta_{i j}$ for $1 \leq i \leq j \leq 3$, and such that for each $u, \mu_{1}(u), \mu_{2}(u)$ and $\mu_{3}(u)$ span the same subspace of $C$ as $\lambda_{1}(u), \lambda_{2}(u)$ and $\lambda_{3}(u)$. Hence $\left\langle\rho, \lambda_{j}(u)\right\rangle=0,1 \leq j \leq 3$, if and only if $\left\langle\rho, \mu_{j}(u)\right\rangle=0,1 \leq j \leq 3$. Therefore it suffices to find a curve $\rho(u)$ in $P$ joining $\rho(0)$ to $\rho(1)$ such that $\left\langle\rho(u), \mu_{j}(u)\right\rangle=0,1 \leq j \leq 3$. For each $v \in[0,1]$ let us pick by Proposition 2.1 an element $\rho_{v} \in P$ such that $\left\langle\rho_{v}, \mu_{j}(v)\right\rangle$ $=0,1 \leq j \leq 3$, and such that $\rho_{0}=\rho(0)$ and $\rho_{1}=\rho(1)$. Let $\rho_{v}(u)=\rho_{v}$ $-\sum_{j=1}^{3}\left\langle\mu_{j}(u), \rho_{v}\right\rangle \mu_{j}(u)$. We see that $\left\langle\rho_{v}(u), \mu_{j}(u)\right\rangle=0$ for $1 \leq j \leq 3$. We also see that there exists a real number $\varepsilon_{v}>0$ depending upon $\rho_{v}$, such that if $|u-v|<\varepsilon_{v}$ then $\rho_{v}(u) \in P$. Let $I_{v}=\left\{u|| u-v \mid<\varepsilon_{v}\right\}$. The $I_{v}$ form an open covering of $[0,1]$, and therefore there exists a finite subcovering $I_{v_{1}} \cdots I_{v_{k}}$. If $I_{0}$ is not in this list throw it in. Hence by relabeling these intervals if necessary, we can find a sequence of intervals $I_{0}, \cdots, I_{k}$, where $I_{n}=I_{v_{n}}$, and points $0=u_{-1}<u_{0}<\cdots<u_{k-1}<u_{k}=1$ with the following properties: $I_{0}=I_{0}, u_{n} \in I_{n} \cap I_{n+1}$, and $1 \in I_{k}$. Therefore $\left[0, u_{0}\right] \subseteq I_{0},\left[u_{0}, u_{1}\right] \subseteq I_{1}, \cdots$, $\left[u_{k-2}, u_{k-1}\right] \subseteq I_{k-1}$ and $\left[u_{k-1}, 1\right] \subseteq I_{k}$. Let $\rho_{n}(u)=\rho_{v_{n}}(u)$ be the curve in $P$ defined by $\rho_{v_{n}}$ on the interval $I_{n}=I_{v_{n}}$, and reparametrize the curves $\mu_{j}(u)$ as follows:

$$
\mu_{j}(w)=\left\{\begin{array}{l}
\mu_{j}\left(u_{n-1}+2\left(w-u_{n-1}\right)\right) \text { for } u_{n-1} \leq w \leq u_{n-1}+\frac{1}{2}\left(u_{n}-u_{n-1}\right) \\
\mu_{j}\left(u_{n}\right) \text { for } u_{n-1}+\frac{1}{2}\left(u_{n}-u_{n-1}\right) \leq w \leq u_{n}, \quad 0 \leq n \leq k .
\end{array}\right.
$$

Let us formally set $\rho_{k+1}\left(u_{k}\right)=\rho_{k+1}(1)=\rho(1)$. We then define $\rho(w)$ by the formulas: 


$$
\begin{aligned}
& \rho_{n}\left[u_{n-1}+2\left(w-u_{n-1}\right)\right] \text { for } u_{n-1} \leq w \leq u_{n-1}+\frac{1}{2}\left(u_{n}-u_{n-1}\right), \\
& \rho_{n}\left(u_{n}\right) \frac{2\left(u_{n}-w\right)}{u_{n}-u_{n-1}}+\rho_{n+1}\left(u_{n}\right) \frac{2 w-u_{n}-u_{n-1}}{u_{n}-u_{n-1}} \\
& \quad \text { for } u_{n-1}+\frac{1}{2}\left(u_{n}-u_{n-1}\right) \leq w \leq u_{n} .
\end{aligned}
$$

Note that the above second formula is a convex sum, and therefore represents a line in $P$ joining $\rho_{n}\left(u_{n}\right)$ to $\rho_{n+1}\left(u_{n}\right)$. Hence $\rho(w)$ is a continuous path in $P$. Finally we see that $\left\langle\rho(w), \mu_{j}(w)\right\rangle=0$ for $1 \leq j \leq 3$ because this is true for $u_{n-1} \leq w \leq u_{n-1}+\frac{1}{2}\left(u_{n}-u_{n-1}\right)$, and because

$$
\left\langle\mu_{j}\left(u_{n}\right), \rho_{n}\left(u_{n}\right)\right\rangle=\left\langle\mu_{j}\left(u_{n}\right), \rho_{n+1}\left(u_{n}\right)>=0 \text { for } 1 \leq j \leq 3\right.
$$

implies

$$
<\mu_{j}\left(u_{n}\right), t \rho_{n}\left(u_{n}\right)+(1-t) \rho_{n+1}\left(u_{n}\right)>=0 \text { for } 0 \leq t \leq 1 \text {, and } 1 \leq j \leq 3 .
$$

This completes our proof.

\section{Bibliography}

[1] E. A. Feldman, The geometry of immersions. I, Trans. Amer. Math. Soc. 120 (1965) $185-224$

[2] W. Fenchel, Uber Krümmung und Windung geschlossener Raumkurven, Math. Ann. 101 (1929) 238-252.

[3] - Geschlossene Raumkurven mit vorgeschriebenem Tangentenbild, Jber. Deutchen Math. Verein 39 (1930) 183-185.

[4] J. R. Munkres, Elementary differential topology, Annal of Math. Studies No. 54, Princeton University Press, Princeton, 1963.

[5] S. Smale, Regular curves on Riemannian manifolds, Trans. Amer. Math. Soc. 87, (1958) 492-512.

[6] N. Steenrod, The topology of fibre bundles, Princeton University Press, Princeton, 1951.

[7] D. J. Struik, Lectures on classical differential geometry, Addison-Wesley, Reading, Mass., 1950.

[8] H. Whitney, On regular closed curves in the plane, Composition Math. 4 (1937) 276-284. 
